\title{
Quality of diet and eating behaviours in children with autism
}

\author{
L. Darvill ${ }^{1}$, E. McCaughey ${ }^{1}$ and S. Wootton ${ }^{2}$ \\ ${ }^{1}$ Wordsworth House, Tremona Road Children's Centre, 121/123 Tremona Road, Southampton SO16 6HU, UK \\ and ${ }^{2}$ Institute of Human Nutrition, University of Southampton, Southampton SO16 6YD, UK
}

\begin{abstract}
Children with autism demonstrate a wider range of more problematic eating behaviours than the general population ${ }^{(1)}$. Although profound shortness, stunting or wasting are not reported, growth may be affected and disordered eating may compromise diet quality and nutrient intake ${ }^{(2)}$. Currently, little is known of the effect of individual eating behaviours on adequacy of dietary intake in children with autism. The present study aimed to better characterize the nature of disordered eating in children with autism and the impact of differences in eating behaviour on the frequency and variety of foods consumed and the range of observed nutrient intake. The study also examined the extent of parental worry about nutritional well-being, reasons contributing to problematic eating behaviours, growth of children with autism and awareness and current practice of healthcare professionals.
\end{abstract}

Fifty children (48\%) were recruited via paediatrician-initiated telephone contact from a community-based cohort of 105 3-11 year olds (mean age 7 years 7 months, forty-four males) with ICD-10 diagnosis of autism ${ }^{(3)}$. Home visits allowed completion of questionnaires including FFQ, $24 \mathrm{~h}$ dietary recall and an adapted brief autism mealtime behaviour inventory (BAMBI) ${ }^{(1)}$. Growth measurements were collected and medical notes reviewed. Senior community paediatricians completed questionnaires assessing their current practice.

BAMBI scores were divided into tertiles representing best, middle-range and worst eating behaviours. A one-way ANOVA was used to analyse differences in mean variety and frequency of food consumed by behaviour tertiles according to FFQ data. The $24 \mathrm{~h}$ recall data were analysed using nutritional analysis software and nutrient intakes were divided into tertiles representing lowest, middle-range and highest consumption. Trends were identified using cross-tabulation to assess the linear-by-linear relationships between the tertiles for behaviour and for specific nutrients.

Problematic eating behaviours are highly prevalent (one-sample $t$ test; $P<0.001$ ) and significantly affect dietary patterns of children with autism. Children with poorer eating behaviours ate a reduced variety of vegetables $(P=0.001$; mean variety $8.4 v .3 .6)$ and protein $(P=0.003$; mean variety $11.2 v .6 .8)$ and consumed less energy overall $(P=0.001$ for trend). Cross-tabulation demonstrated significant trends towards lower intakes of key micronutrients in those with poorest eating behaviours; the linear-by-linear association (as $P$ for trend) between worsening behaviour score (highest BAMBI tertiles) and lower tertiles of intakes of $\mathrm{Fe}(0.02), \mathrm{Zn}(0.011), \mathrm{Ca}(0.011)$, riboflavin (0.003), vitamin A (0.02) and fibre (0.036). Macronutrient intakes, however, were unrelated to behaviour.

Poor eating behaviours correlated positively with increased parental worry about balance of diet $(r 0.53, P<0.001)$. Behaviours representing 'limited variety' were most problematic for parents, largely for perceived 'nutritional reasons'. Growth was above average (mean height SD score 0.73 , weight SD score 0.80 ), and largely unaffected by adverse behaviour patterns, although consideration may be directed at the risk of weight gain as the mean BMI SD score was 0.78. Professional advice and guidance to manage autism-related nutritional issues could be improved.

Children with autism are at increased risk of adverse eating behaviours, which negatively impact on food choice and the balance of their diets. Objective screening for children with poorest eating behaviours is important clinically in order to identify those at risk for poor nutrition and social implications of problematic behaviours. The study findings support the need to effectively identify those most at risk, with subsequent development of targeted guidelines and specialist services to manage the very unique behavioural and nutritional needs of children with autism in clinical practice.

1. Schreck K, Williams K \& Smith (2004) J Autism Dev Dis 34, 433-438.

2. Cornish E (1998) J Hum Nutr Dietet 11, 501-509.

3. World Health Organization (2007) International Classification of Diseases, 10th ed. Geneva: WHO.

4. Lukens C \& Linscheid T (2008) J Autism Dev Disord 38, 342-352; Epublication 20 June 2007. 